\title{
Ellipsometry Data Analysis and Ellipsometric Spectra of Complex Materials
}

\author{
DANKA B. STOJANOVIĆ, University of Belgrade, \\ Vinča Institute of Nuclear Sciences, \\ Laboratory of Atomic Physics, Belgrade \\ JELENA V. RADOVANOVIĆ, University of Belgrade, \\ School of Electrical Engineering, Belgrade \\ VITOMIR B. MILANOVIĆ, University of Belgrade, \\ School of Electrical engineering, Belgrade \\ ZLATKO LJ. RAKOČEVIĆ, University of Belgrade, \\ Vinča Institute of Nuclear Sciences, \\ Laboratory of Atomic Physics, Belgrade
}

\author{
Original scientific paper \\ UDC: $535.51: 66.017 / .018$
}

Optical and structural properties of materials can be characterized by spectroscopic ellipsometry which represents an experimental technique that measures polarized light reflected from a material surface. Since the most complicated part of this method is data analysis and modeling, we present the calculation of the ellipsometric parameters for bi-isotropic materials. We consider the ellipsometric model for these materials which require not just permittivity like in conventional model, but also magnetic permeability and magnetoelectric coupling.

Key words: ellipsometry, optical properties, bi-isotropic materials, Tellegen media

\section{INTRODUCTION}

Optical characterization of the materials is an important topic in the research area of electromagnetism. Ellipsometry is such a characterization technique which is based on measurement of the changes in the state of polarization of light upon light reflection from a surface. When light is reflected from the sample at oblique incidence, it is classified into p- and s-polarized light waves. Whether the wave is $\mathrm{s}$ or $\mathrm{p}$ polarized depends on the oscillatory direction of its electric field and each light wave shows different behavior. Ellipsometry measured values, $\Delta$ and $\Psi$, are the phase difference and ratio of amplitudes of $\mathrm{p}$ and $\mathrm{s}$ polarizations, respectively [1]. For getting useful information from the ellipsometric measurements, we are usually using ellipsometric software. However, for characterization of some materials ellipsometric software is not sufficient and numerical calculation of the model is necessary.

During recent decades, a variety of novel and co-

Author's address: Danka Stojanović, University of Belgrade, Vinča Institute of Nuclear Sciences, Laboratory of Atomic Physics, Belgrade, Mike Alasa 12-14

Paper received: 17.01.2014.

Paper accepted: 27.02.2014. mplex materials have been designed. For the description of the relations between electric and magnetic fields for some materials, standard constitutive equations cannot be used. In the case of bi-isotropic media, there is an additional factor in the constitutive relations due to magnetoelectric coupling. Because of this effect, these materials are called bi-isotropic which means that there are two exciting fields and two response flux densities [2]. In this study, we investigated Tellegen material, which is a subclass of biisotropic materials. The Tellegen particles are considered as particles with permanent electric and magnetic moments. The existence of such a particle was the theme of many discussions although there are some experimental evidences $[3,4,5]$ as well. One of the solutions was an artificial Tellegen particle which was made of a small ferrite sphere and a small piece of a thin metal wire sealed to a sphere [3]. The other was quasi-two-dimensional ferrite disk with magnetic-dipolar-mode oscillations which has magnetoelectric properties [4]. In reference 5 are presented Janus particles which are considered as the Tellegen particles with permanent electric and magnetic moments. The system consists of ferromagnetic dopant added to a stream of $100 \mu \mathrm{m}$ spherical polyethylene particles [5]. 
In this work, we calculated reflection coefficients and ellipsometric parameters for bi-isotropic Tellegen materials. An interesting result is the cross-polarization reflection spectrum. Cross-polarization appears as a consequence of rotation of reflected wave [2]. Since the ellipsometric measurements are dependent on incident angle, we present reflection coefficients as a function of incidence angle for different values of magnetoelectric coupling. From these spectra, we can estimate the value of Brewster's angle. The sensitivity of the measurement increases, and the ratio of reflections for $\mathrm{p}$ and $\mathrm{s}$ polarizations is maximized at this angle [1]. In addition, we have evaluated the spectra of reflection coefficients for a fixed value of incident angle.

\section{ELECTROMAGNETIC WAVE IN TELLEGEN MEDIA}

The constitutive equations for bi-isotropic materials are:

$$
\begin{aligned}
& \mathbf{D}=\varepsilon \mathbf{E}+\xi \mathbf{H} \\
& \mathbf{B}=\zeta \mathbf{E}+\mu \mathbf{H}
\end{aligned}
$$

where $\mathbf{D}$ is dielectric displacement, $\mathbf{E}$ is electric field, $\mathbf{B}$ is magnetic induction and $\mathbf{H}$ is magnetic field, $\varepsilon$ is permittivity, $\mu$ is permeability and $\xi$ and $\zeta$ are magnetoelectric parameters. In the most general case they are presented with:

$$
\begin{aligned}
& \xi=\frac{\chi-j \kappa}{c} \\
& \zeta=\frac{\chi+j \kappa}{c}
\end{aligned}
$$

where $\kappa$ is the chirality, a dimensionless parameter which describes the handedness of the material and $\chi$ is the Tellegen parameter, a dimensionless quantity which represents the degree of nonreciprocity in the medium [6]. Here, we focus on non-reciprocal Tellegen medium which corresponds to the case $\kappa=0$. Coupling between electric and magnetic fields is described with Maxwell equations:

$$
\begin{aligned}
& \nabla \times \mathbf{E}=j \omega \mathbf{B} \\
& \nabla \times \mathbf{H}=-j \omega \mathbf{D}
\end{aligned}
$$

From equations (1), (2), (5) and (6) we obtain the wave equations for the case of $\mathrm{s}$ and $\mathrm{p}$ polarization, respectively:

$$
\begin{aligned}
& \nabla \times\left(\left(\mu^{-1}\right)(\nabla \times \mathbf{E})\right)+\omega^{2} \chi^{2}\left(\mu^{-1}\right) \mathbf{E}-\omega^{2} \varepsilon \mathbf{E}=0 \\
& \nabla \times\left(\left(\varepsilon^{-1}\right)(\nabla \times \mathbf{H})\right)+\omega^{2} \chi^{2}\left(\varepsilon^{-1}\right) \mathbf{H}-\omega^{2} \mu \mathbf{H}=0
\end{aligned}
$$

From these equations we get the dispersion relation:

$$
k=\sqrt{\omega^{2}\left(\varepsilon \mu-\chi^{2}\right)-k_{p a r}^{2}}
$$

where $k_{0}=\omega / c$ represents the wave vector in air and $k_{\text {par }}=k_{0} \sin \theta$ is the parallel component of the wave vector, while $\theta$ is the angle of incidence.

\section{FRESNEL REFLECTION COEFFICIENTS AND ELLIPSOMETRIC PARAMETERS}

In order to determine the reflection coefficients, we consider the boundary conditions for interface of air and Tellegen medium (Figure 1, where air is white and Tellegen media is light-blue). Magnetoelectric effect of this medium has an influence on the reflection of a plane wave [2]. When a linearly polarized incident wave is reflected from this medium, the reflected wave is also linearly polarized but it has a different direction. Due to this effect of cross-polarization [2], the Fresnel reflection coefficients are represented with a matrix with four elements $\left(r_{s s}, r_{s p}, r_{p s}, r_{p p}\right)$ :

$$
\begin{aligned}
& r_{s s}=\frac{u v+u-v-1}{1+u+v+u v} \\
& r_{s p}=\frac{-2 \chi u Z_{0} / \mu_{2}}{1+u+v+u v},
\end{aligned}
$$$$
r_{p s}=\frac{-\chi}{\varepsilon_{2}\left(u+Z_{0}\right)}\left(\frac{2 \varepsilon_{2} \mu_{2}\left(1+Z_{0} u\right)}{\varepsilon_{2} \mu_{2}(1+v)\left(1+Z_{0} u\right)-Z_{0} \chi^{2} v u}\right) \text { (12) }
$$$$
r_{p p}=1-\frac{2 \varepsilon_{2} \mu_{2}\left(1+Z_{0} u\right)}{\varepsilon_{2} \mu_{2}(1+v)\left(1+Z_{0} u\right)-Z_{0} \chi^{2} v u}
$$

where $\quad u=k_{1} \mu_{2} / k_{2} \mu_{1}, \quad v=k_{1} \varepsilon_{2} / k_{2} \varepsilon_{1} \quad$ and $Z_{0}=\sqrt{\mu_{0} / \varepsilon_{0}}$.

Permittivities and permeabilities of air and biisotropic material are, respectively, $\varepsilon_{1}=1, \mu_{1}=1$ and $\varepsilon_{2}=10, \mu_{2}=2$.

a)
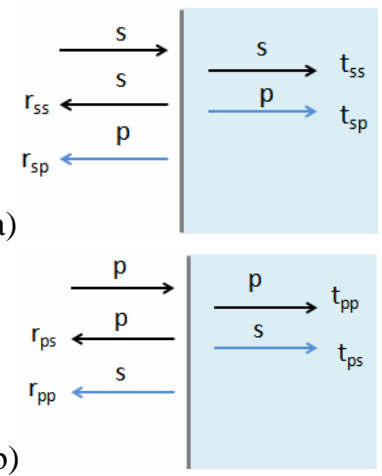

Figure 1-Model of scattering of incident wave in the case of: a) s polarized incident wave $b$ ) $p$ polarized incident wave. 
As we can see from figures 2 and 3, the value of the Tellegen parameter $\chi$ influences the amplitudes of reflection coefficients $r_{s s}$ and $r_{p p}$. The value of the angle of incidence is fixed at $\theta=70^{\circ}$. It is not only that this parameter influences on the reflection, but at the same time the reflection helps us to estimate the value of Tellegen factor $\chi$ [7]. The values of $\chi$ span from 0 to $10^{-8}$ and values from this range are used in all the figures in this work (table 1).

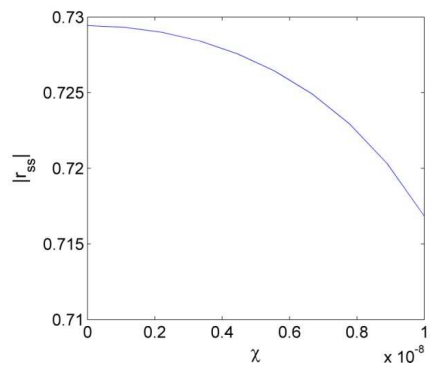

Figure 2 - Amplitude of the reflection coefficient $r_{s s}$ as a function of $\chi$.

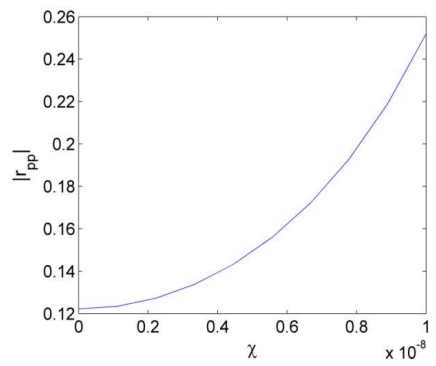

Figure 3 - Amplitude of the reflection coefficient $r_{p p}$ as a function of $\chi$.

From figure 2 we observe that reflection coefficient $r_{s s}$ decreases as $\chi$ increases. On the contrary, regarding $r_{p p}$, higher values of reflection coefficient correspond to increase in $\chi$ (Figure 3).

Table 1. Values of parameter $\chi$ used in the following spectra (for figures 4, 5, 6, 7, 8).

\begin{tabular}{|l|l|}
\hline $\begin{array}{l}\text { The color of spectral } \\
\text { line }\end{array}$ & $\chi$ \\
\hline & 0 \\
\hline & $1.1 * 10^{-9}$ \\
\hline & $2.2 * 10^{-9}$ \\
\hline & $3.3 * 10^{-9}$ \\
\hline & $4.4 * 10^{-9}$ \\
\hline & $5.5 * 10^{-9}$ \\
\hline & $6.6 * 10^{-9}$ \\
\hline & $7.7 * 10^{-9}$ \\
\hline & $8.8 * 10^{-9}$ \\
\hline & $1 * 10^{-8}$ \\
\hline &
\end{tabular}

As far as the dependence of reflection coefficients $r_{s s}$ and $r_{p p}$ on the incidence angle is concerned, we can conclude that the change of spectra happens at Brewster's angle (Figure 4 and Figure 5). The Brewster's angle is approximately in the range of $55^{\circ}$ $65^{\circ}$, depending on the value of $\chi$ (table 1 ). In the case of $r_{s s}$, until $\theta$ reaches this point, $r_{s s}$ is higher for higher $\chi$, but after this value of $\theta$, the characteristic is the opposite.

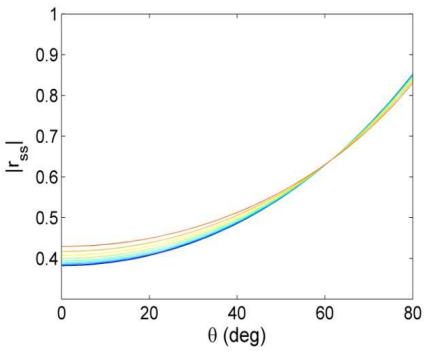

Figure 4 - Amplitude of the reflection coefficient $r_{s s}$ as a function of $\theta$ for different values of $\chi$.

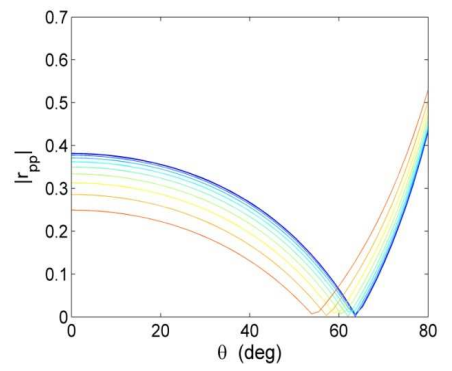

Figure 5 - Amplitude of reflection coefficient $r_{p p}$ as a function of $\theta$ for different values of $\chi$.

On the other side, for smaller angles, the value of $r_{p p}$ is higher for lower $\chi$, but for the angles higher than the Brewster's angle, the characteristic becomes the opposite. Also, we can observe that $r_{p p}$ is zero for Brewster's angle. This result is the same in the case of standard material without magnetoelectric coupling effect. The zero coefficient of reflection for $\mathrm{p}$ polarisation for this angle is the consequence of electric dipole radiation at the interface [1].

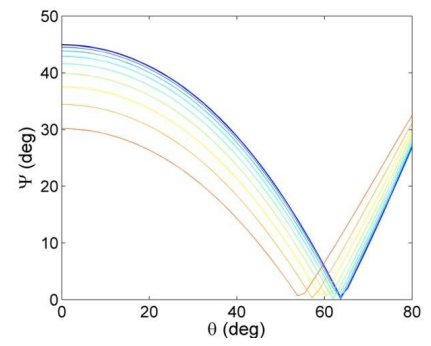

Figure 6 - Ellipsometric angle $\psi$ as a function of incidence angle $\theta$ for different values of $\chi$. 
In addition, we investigated the influence of the value of angle of incidence $\theta$ on ellipsometric $\psi$ parameter for different values of $\chi$ (figure 6). Parameter $\psi$ is defined as:

$$
\tan \Psi=\frac{\left|r_{p p}\right|}{\left|r_{s s}\right|}
$$

We can conclude that for lower values of Tellegen parameter $\chi$ we get higher values of angle $\psi$. With regard to $\theta$, we observe the similar behaviour, if incidence angle is higher, then the magnetoelectric coupling is lower. These statements are valid until $\theta$ reaches the Brewster's angle, but after this point the characteristic is the opposite.

When it comes to cross-polarized waves, the reflection coefficients $r_{s p}$ and $r_{p s}$ increase for increasing Tellegen parameter $\chi$ (Figures 7 and 8 , respectively). This is an expected result since $r_{s p}$ and $r_{p s}$ are the direct consequence of magnetoelectric effect in this medium. From these results we conclude that in the case of $\mathrm{p}$ polarization, for Brewster's incident angle, the only reflection which exists in the media is cross polarized reflection- $r_{p s}$.

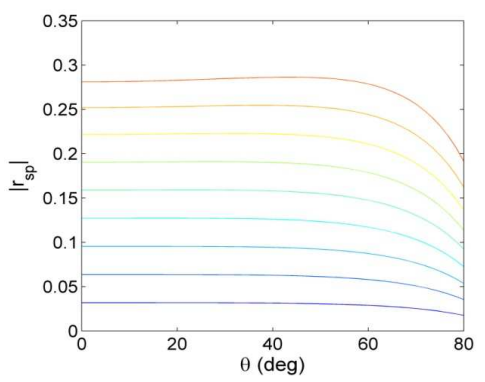

Figure 7 - Amplitude of the reflection coefficient $r_{s p}$ as a function of $\theta$ for different values of $\chi$.

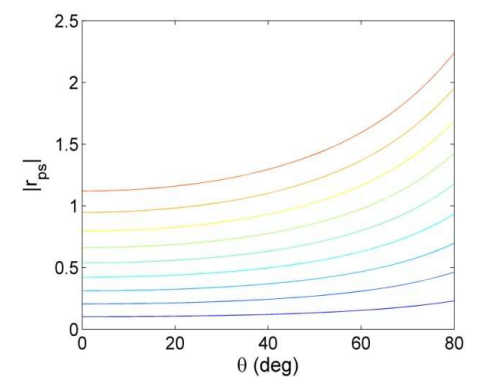

Figure 8 - Amplitude of the reflection coefficient $r_{p s}$ as a function of $\theta$ for different values of $\chi$.

Further investigation of these materials should be continued with magneto-optical Kerr effect microscopy-MOKE. Similarly, in the case of MOKE, solving of Maxwell's equations leads to magneto-optical Fre- snel reflection coefficients [8]. In this method, the influence of rotation of the plane of polarized light on reflection is magnetization dependant. This rotation is converted into a domain contrast which can be seen after image processing [9].

\section{CONCLUSION}

Among various advantages of spectroscopic ellipsometry, very important one is a wide range of materials for application. Because of the complexity of some materials, additional modeling and data analysis of the results of the ellipsometric measurement is required. Here we presented Tellegen bi-isotropic media, as an example for studying optical properties and ellipsometric data analysis of complex materials. We investigated the influence of the incidence angle on the reflection amplitude for different values of magnetoelectric coupling. For $\mathrm{s}$ polarized or $\mathrm{p}$ polarized waves, the spectral characteristic become different after reaching Brewster's angle. With regard to crosspolarized waves, we can observe that there is a decrease in reflection coefficients with decrease of Tellegen parameter. In the case of $\mathrm{p}$ polarization, for Brewster's incidence angle, there is only cross-polarized reflection. Also, we presented spectra for ellipsometric parameter $\psi$ as a function of magnetoelectric coupling and incidence angle.

\section{REFERENCES}

[1] H. Fujiwara, Spectroscopic ellipsometry, Wiley, Tokyo, Japan, 2007, p.1-10.

[2] S. Zouhdi, A. Shivola, A. Vinogradov, Metamaterials and Plasmonics: Fundaments, Modelling, Applications, Springer, Dordrecht, The Netherlands, 2008, p.16-18.

[3] S. Tretyakov, S. Masovski, I. Nefedov, P. Belov, A. Sanmartin, Electromagnetics 23, 665-680, 2003.

[4] E. O. Kamenetskii, M. Sigalov, and R. Shavit J. Appl. Phys. 105, p. 013537-01-14, 2009.

[5] A. Ghosh, N. K. Sheridon, and P. Fischer, e-print arXiv:0708.1126.

[6] I. V. Lindell, A. H. Sihvola, S. A. Tretyakov, and A. J. Viitanen, Electromagnetic Waves in Chiral and Bi-Isotropic Media, Artech House, Boston, MA, 1994.

[7] A. Sihvola, S. Tretyakov, Optik 119, p. 247-249, 2008.

[8] C-Y. You, S-C Shin, J. Appl. Phys. 84, p. 541-546, 1998.

[9] R. Schafer, Handbook of Magnetism and Advanced Magnetic Materials: Investigation of domains and dynamics of domain walls by the magneto-optical Kerr-effect, Volume 3, John-Wiley and Sons, 2007. 


\section{REZIME}

\section{ANALIZA PODATAKA DOBIJENIH ELIPSOMETRIJOM I ELIPSOMETARSKI SPEKTAR KOMPLEKSNIH MATERIJALA}

Cilj ovog rada je da se prikaže kako se može napraviti model $i$ uraditi analiza podataka dobijenih spektroskopskom elipsometrijom u slučaju kompleksnih materijala. Kao primer za izračunavanje optičkih osobina materijala $i$ elipsometarskih parametara korišćeni su bi-izotropni materijali. Magnetoelektrično sprezanje u ovim materijalima utiče na rasejanje incidentog talasa što se primećuje u spektrima amplituda refleksije i elipsometarskog parametra $\Psi$. Predstavljeni su i spektri refleksije unakrsno-polarisanih talasa u zavisnosti od ugla incidencije za različite vrednosti magnetoelektričnog sprezanja.

Ključne reči: elipsometrija, optičke osobine materijala, bi-izotropni materijali, Telegen medijum 